\title{
Otogenic Brain Abscess Presenting with Gait Ataxia
}

\author{
Sang Huck Cho ${ }^{1}$, Moo Kyun Park ${ }^{1}$, Jong Dae Lee ${ }^{1}$ and Sun Chul Hwang ${ }^{2}$ \\ ${ }^{1}$ Departments of Otorhinolaryngology-Head and Neck Surgery, ${ }^{2}$ Neurosurgery, College of Medicine, Soonchunhyang University, \\ Bucheon, Korea
}

Received October 4, 2011

Revised March 11, 2012

Accepted March 15, 2012
Although the occurrence of otogenic brain abscess has been considerably reduced through improvements to antibiotics, brain abscesses remains one of the most significant life threatening complications of chronic otitis media. We report the case of a 67 -year-old male patient who presented with gait ataxia and dizziness. Imaging studies revealed a left cerebellar abscess and extensive destructive changes to the labyrinth due to chronic otitis media. We conclude that otogenic brain abscess should be considered as differential diagnosis in patients with chronic otitis media who develop central vertigo.

Korean J Audiol 2012;16:31-34

\section{Introduction}

Central dizziness is mainly divided into vascular and nonvascular causes, the most common of which is vertebrobasilar ischemic stroke. Among the non-vascular causes, cerebellopontine tumors such as acoustic tumor are the most prevalent. ${ }^{1,2)}$ In cases in which gait ataxia is accompanied with dizziness, a cerebellum invading central disorder can be suspected.

Most abscess that occurs in the cerebellum are otogenic brain abscesses. ${ }^{3)}$ Although the frequency of these, which occur as a complication of otitis media, especially cholesteatoma, has dramatically decreased with advancements in overall antibiotic treatment and diagnostic imaging technology such as CT, MRI, and others, the disease can still lead to death unless properly treated. It is therefore important to fully understand the early symptoms and clinical manifestations and perform precise diagnosis and treatment. ${ }^{4)}$ General symptoms of cerebellar abscess are right gait ataxia and abnormal manifestations that are indicated on a cerebellar function test.

This study details a patient who was admitted with gait ataxia and dizziness caused by a cerebellum abscess that, occurred as a complication of chronic otitis media as chief complaints, which is reported it together with a literature review.

\section{Case Report}

A 67 year old male patient was admitted to the emergency room with dizziness and gait ataxia that had suddenly developed two days previously. The patient was receiving pharmacological treatment for hypertension and diabetes and there was no relevant family history. Other than the dizziness and gait ataxia, the patient complaining of headache, hearing loss and aural pressure in the left ear, and aural discharge but these did not accompany otalgia or tinnitus. The patient displayed a clear state of consciousness in neurological examination, his papillary reflex was normal, and nystagmus was not observed. The patient fell toward left even with his eyes open in the Romberg test and displayed dysmetria on the left side in the cerebellar function finger to nose test. Brain CT taken in the emergency room displayed the formation of a common wall, including the wall that enhanced the contrast in a circle of $5 \times 5 \mathrm{~cm}$ toward the left cerebellum and a manifestation in which it pressed the brain stem and the fourth ventricle (Fig. 1A). With the suspicion of brain abscess in the left cerebellum, the patient was admitted to the neurosurgical ward and received antibiotic treatment. Two days after he was admitted to hospital, his state of consciousness suddenly altered to drowsy and craniotomy and drainage of abscess was performed in response. As a re- 

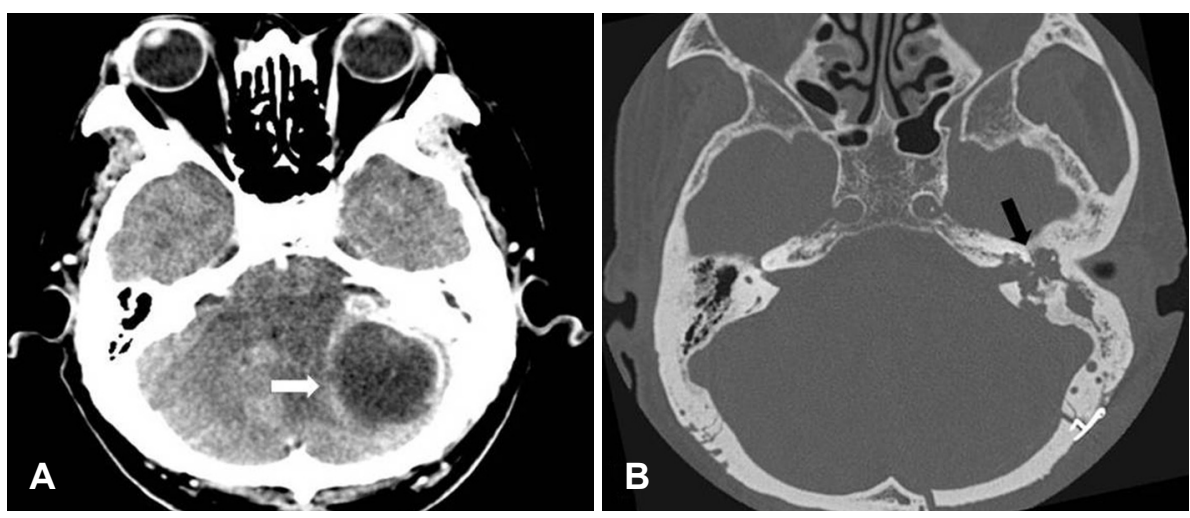

Fig. 1. Preoperative imaging studies. A: Brain CT shows a low signal density lesion with rim enhancement in the left cerebellar hemisphere (white arrow). B: Temporal bone CT after abscess drainage shows extensive destruction of the petrous bone including cochlear and semicircular canals (black arrow).
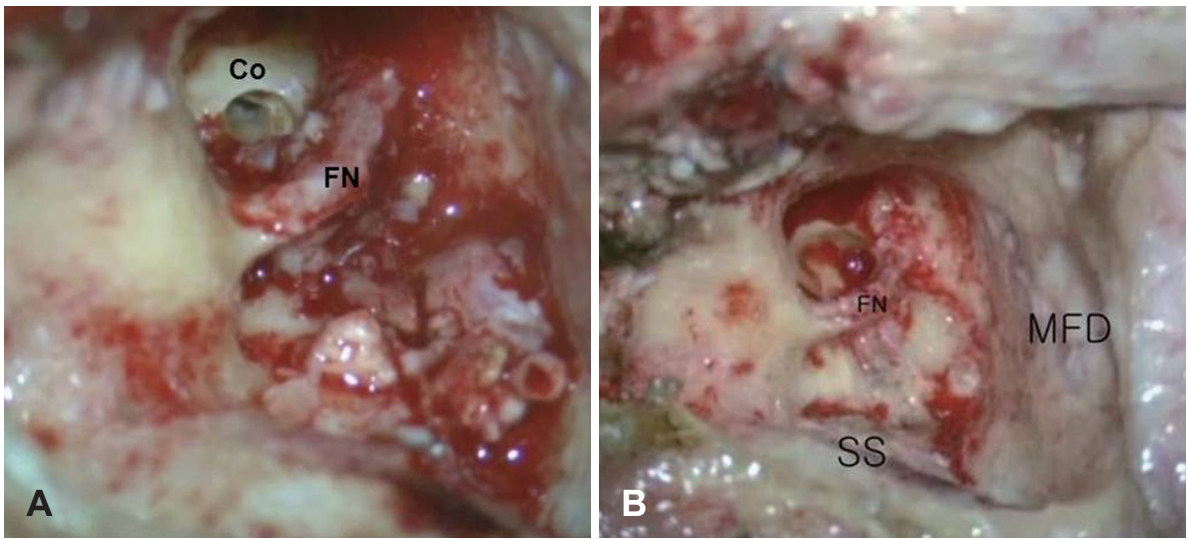

Fig. 3. Surgical findings. A: Cholesteatoma and granulation tissue was found in the mastoid cavity infiltrating the labyrinth, middle ear and cochlea. $\mathrm{B}$ : The lesions were removed via transotic approach. FN: facial nerve, Co: cochlear, MFD: middle fossa dura, SS: sigmoid sinus.

sult of requesting department of otorhinolaryngology for physical examination in order to assess the causative diseases after surgery, it was found that the external auditory canal was narrow and aural discharge and granulation tissue were observed in the left ear. The right ear was normal. Tympanic membrane retraction and the aural discharge that accompanies keratin were also observed (Fig. 2).

On temporal bone CT taken with the suspicion of left otitis media cholesteatomatica, an overall destruction of the cochlea and vestibular organ in the left ear caused by cholesteatoma was indicated (Fig. 1B). Air conduction hearing and bone conduction hearing of $36 \mathrm{~dB}$ respectively was presented for the right ear and hearing loss was found in the left ear when performing pure tone audiometry. The degree of paralysis in the left semicircular duct was $100 \%$ on the results of a caloric test using air. Under diagnosis of otitis media cholesteatomatica, which causes cerebellar abscess with overall invasion of labyrinth and intracranial expansion, cholesteatoma and granulation tissue were removed through transotic approach (Fig. 3). The surgery was performed a month after performing drainage of the abscess in neurosurgery. As the granulation tissue including keratin had infiltrated the mastoid antrum, auris media, and vestibular labyrinth and had destructed the bone structure, it was difficult to pinpoint the landmark that is seen during

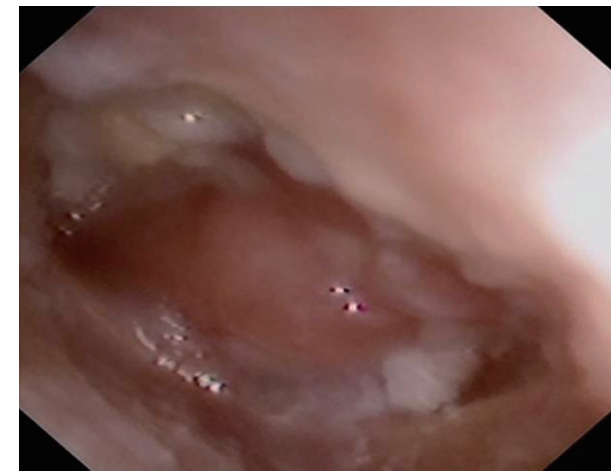

Fig. 2. Photograph of the left ear shows adhesive, wet eardrum with granulation tissue and keratinous material.

tympanomastoidectomy. Although the abscess was discharged within the cochlea, there was invasion into the internal acoustic canal. The junction between the mastoid antrum and posterior fossa was cut after connecting with fibrous tissue and there was no outflow of cerebrospinal fluid. As partial outflow of cerebrospinal fluid was found in the internal acoustic canal, this was stopped with soft tissue and the surgery was finished after filling the deletion by sampling fatty tissue from the stomach. As a result of assessing the bacterial culture from the abscess that was displayed in the mastoid antrum during surgery, group D enterococcus was cultivated and the result for acid- 


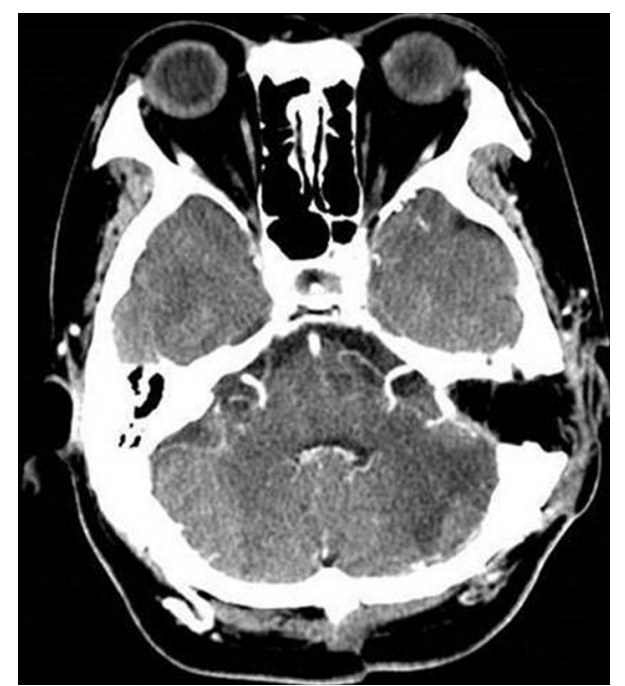

Fig. 4. Brain CT at 6 months after surgery shows improved lesion.

fast bacilli culture was negative. The patient was discharged from the hospital after recovery without neurological sequela and is currently under observation through follow-up CT without relapse (Fig. 4).

\section{Discussion}

Otitis media, unless properly treated, may cause intracranial complication as the inflammation of the auris media caused by the infection spreads inside the cranial cavity. Although the prevalence has dramatically decreased with improved use of antibiotics, it still presents a high mortality rate. ${ }^{5)}$

Otogenic brain abscess mainly develops in the temporal lobe and cerebellum and it accounts for half of all brain abscesses and most commonly occurs as a complication of otitis media cholesteatomatica. In general, brain abscesses that develop in the temporal lobe are more common than those that develop in the cerebellum. ${ }^{6}$ However, cerebellum abscess is known to be particularly serious as the condition is difficult to diagnose with only clinical diagnosis and drainage is likely to be insufficient. Also, it presents higher mortality rate and is likely to be multicentric. A brain abscess in the temporal lobe develops as direct bone erosion, whereas a brain abscess in the cerebellum develops due to thrombophlebitis.

Complaints of dizziness caused by otitis media are mainly divided into case in which labyrinthine fistula and labyrinthitis are present and there is invasion into the labyrinth as an intratemporal complication and cases in which it invades into the cerebellum as an intracranial complication. ${ }^{7,8)}$ The patient in this study visited hospital with symptoms of central vestibular nystagmus including gait ataxia, and others as otitis media cholesteatomatica invaded the posterior cranial fossa and caused a cerebellum abscess.

Otogenic brain abscess is accompanied by headache, aural symptoms including aural discharge in the left ear, nausea, emesis, fever, and others. ${ }^{9)}$ Also, the general local symptoms of cerebellum abscess are right ataxia, vertical or circular spontaneous nystagmus with alteration, weakness in right muscles, and incoordination. ${ }^{10)}$ Due to pressure on the brain stem, intermittent bradysphygmia is frequently present and increases in blood pressure, extensive change in body temperature, and others symptoms are also observed. In the patient of this case study, headache, aural discharge in the left ear, and right ataxia were present.

In the beginning stage of treatment, it is important to stabilize the patient similarly as for encephalomeningitis, to treat the active inflammation by detecting primary lesion such as cholesteatoma, and to take measures regarding the increase in intracranial pressure and potential seizure. Intravenous administration of antibiotics is the main treatment for brain abscess. Antibiotics are administered for several weeks and follow-up recording the change in abscess should be carried out by regular CT. Since primary lesion cannot be resolved with the administration of antibiotics alone, any cholesteatoma and granuloma remaining in the mastoid should be surgically removed. ${ }^{11,12)}$

Although there is some controversy regarding the order of treatment, it is generally best to perform neurosurgery such as aspiration, drainage surgery, and others with clear surgical vision after full administration of a vast quantity of antibiotics in order to first treat the brain abscess and then perform radical operation of the middle ear after the condition of the patient has improved with time. ${ }^{3)}$ However, a method involving direct drainage of the brain abscess within the mastoid while performing tympanomastoidectomy has also been performed. ${ }^{4)}$

With regards to the patient in this study, the ear was not assessed in the initial examination but the patient was then transferred to our department with the suspicion of otitis media after X-ray testing. In general, when examining patients with the complaints of dizziness, it is important to examine the ear and perform hearing tests in order to determine whether or not there is secondary dizziness from otitis media. It is also necessary to keep in mind the links between ear diseases and testing of the head and brain in patients who show neurological symptoms. It is also important to use proper antibiotics in order to prevent complications from otitis media and perform necessary treatment in case of the occurrence of complications when otitis media is diagnosed.

\section{REFERENCES}

1) Lee H. Neuro-otological aspects of cerebellar stroke syndrome. J Clin Neurol 2009;5:65-73. 
2) Traccis S, Zoroddu GF, Zecca MT, Cau T, Solinas MA, Masuri R. Evaluating patients with vertigo: bedside examination. Neurol Sci 2004;25 Suppl 1:S16-9.

3) Erdoğan E, Cansever T. Pyogenic brain abscess. Neurosurg Focus 2008;24:E2.

4) Dubey SP, Larawin V. Complications of chronic suppurative otitis media and their management. Laryngoscope 2007;117:264-7.

5) Osma U, Cureoglu S, Hosoglu S. The complications of chronic otitis media: report of 93 cases. J Laryngol Otol 2000;114:97-100.

6) Nunez DA, Browning GG. Risks of developing an otogenic intracranial abscess. J Laryngol Otol 1990;104:468-72.

7) Gersdorff MC, Nouwen J, Decat M, Degols JC, Bosch P. Labyrinthine fistula after cholesteatomatous chronic otitis media. Am J Otol
2000;21:32-5

8) Dubey SP, Larawin V, Molumi CP. Intracranial spread of chronic middle ear suppuration. Am J Otolaryngol 2010;31:73-7.

9) Bradley PJ, Manning KP, Shaw MD. Brain abscess secondary to otitis media. J Laryngol Otol 1984;98:1185-91.

10) Miyamoto RT, Worth RM. Otogenic cerebellar abscess. Ann Otol Rhinol Laryngol 1986;95(6 Pt 1):647-8.

11) Penido Nde O, Borin A, Iha LC, Suguri VM, Onishi E, Fukuda Y, et al. Intracranial complications of otitis media: 15 years of experience in 33 patients. Otolaryngol Head Neck Surg 2005;132:37-42.

12) Wanna GB, Dharamsi LM, Moss JR, Bennett ML, Thompson RC, Haynes DS. Contemporary management of intracranial complications of otitis media. Otol Neurotol 2010;31:111-7. 